\title{
Superheavy Nuclei and Related Phenomena
}

\author{
K. P. Santhosh ${ }^{1}$, C. Nithya ${ }^{1}$ \\ ${ }^{I}$ School of Pure and Applied Physics, Kannur University, Swami Anandatheertha Campus, Payyanur 670 327, \\ Kerala, India
}

\begin{abstract}
An overview of decay modes of superheavy nuclei, the proton decay, the alpha decay, the cluster decay and the spontaneous fission, have been studied by considering the isotopes of darmstadtium $\quad(Z=$ 110 ) within the range $256 \leq A \leq 275$. It is seen that the isotopes ${ }^{256-262} D$ s are proton emitters. The proton decay half-lives were calculated using the Gamow like model. Alpha decay half-lives and cluster decay half-lives were calculated using the Coulomb and proximity potential model (CPPM). Alpha decay half-lives were also calculated using Viola-Seaborg semi-empirical relationship, Universal cure of Poenaru et al., analytical formula of Royer et al., and the Universal decay law for a theoretical comparison. Spontaneous fission halflives were evaluated using the new shell-effect-dependent formula proposed by Santhosh et al. The semiempirical formula of Xu et al., formula proposed by Bao et al., and the formula of Ren et al., have been also used for calculating the spontaneous fission half-lives. From our study it is seen that most of the superheavy nuclei are prone to proton decay, the alpha decay, the cluster decay and the spontaneous fission.
\end{abstract}

Keywords: Proton decay, Alpha decay, Cluster decay, Spontaneous fission, Superheavy elements.

\section{Introduction}

The studies of physical as well as chemical properties of superheavy nuclei have received much attention in recent years. The quest for superheavy nuclei began in 1960s with the prediction of island of stability $[1,2]$. Recently the isotopes of many superheavy nuclei have been synthesized using hot fusion reaction [3] performed at JINR FLNR (Dubna) and cold fusion reaction [4] performed at RIKEN (Japan) and GSI (Darmstadt, Germany).

Understanding of decay modes and half-lives are very important in the study of superheavy nuclei. Most of the isotopes of superheavy nuclei discovered so far were identified by observing their radioactive decay chain. The decay modes of superheavy nuclei includes proton decay, alpha decay, spontaneous fission and cluster decay. Theoretically all these emissions have the same underlying mechanism in Physics, the quantum tunneling effect. Superheavy nuclei are proton rich nuclei and hence exiting decay modes such as two proton emission may be observable. Nuclei lying above the proton drip line with positive disintegration energy are proton unstable and decay through proton emission. Up to now several attempts [5-7] have been made to explain the phenomenon of proton decay. Alpha decay and spontaneous fission are the two dominant decay modes of superheavy nuclei. Superheavy nuclei prominently exhibit sequential alpha decay chains followed by spontaneous fission. Alpha decay from superheavy nuclei are extensively studied using various theoretical models [8-11] since it is considered as the direct evidence for the existence of superheavy nuclei. The complexity in the fission process and uncertainty in the nature of fission make spontaneous fission half-lives of superheavy nuclei uncertain. Many theoretical formalisms [12-15] are also there for calculating the spontaneous fission half-lives. The emission of clusters heavier than alpha particle is also relevant in the region of superheavy nuclei $[16,17]$.

In this work we are evaluating the various decay modes of superheavy nuclei Ds within the range 256 $\leq \mathrm{A} \leq 275$ including proton emission, alpha decay, spontaneous fission and cluster decay. Alpha decay and cluster decay half-lives are calculated using the Coulomb and proximity potential (CPPM) proposed by Santhosh et al [18]. A brief outline of CPPM is presented in Section 2. The results and discussion is given in Section 3. A brief summary of the results is given in the last section.

\section{Coulomb and proximity potential model (CPPM)}

In CPPM the interacting potential between two nuclei is taken as the sum of Coulomb potential, proximity potential and centrifugal potential, for both the touching configuration and for the separated fragments. For the pre-scission (overlap) region, simple power law interpolation has been used.

The interacting potential barrier for two spherical nuclei is given by:

$$
V=\frac{Z_{1} Z_{2} e^{2}}{r}+V_{p}(z)+\frac{\hbar^{2} \ell(\ell+1)}{2 \mu r^{2}} \text { for } z>0
$$

National Conference on Current Advancements in Physics $3^{\text {rd }} \& 4^{\text {th }}$ February 2017 
Here $Z_{1}$ and $Z_{2}$ are the atomic numbers of the daughter and emitted cluster, ' $r$ ' is the distance between fragment centres, ' $z$ ' is the distance between the near surfaces of the fragments, $\ell$ represents the angular momentum and $\mu$ the reduced mass. $V_{p}$ is the proximity potential given by Blocki et al., [19, 20] as:

$$
V_{p}(z)=4 \pi j\left[\frac{C_{1} C_{2}}{\left(C_{1}+C_{2}\right)}\right] \Phi\left(\frac{z}{b}\right)
$$

With the nuclear surface tension coefficient:

$$
\gamma=0.9517\left[1-1.7826(N-Z)^{2} / A^{2}\right] \mathrm{MeV} / \mathrm{fm}^{2}
$$

Here $N, Z$ and $A$ represent the neutron, proton and mass number of the parent nuclei. $\Phi$ represents the universal proximity potential [20] given as:

$$
\begin{aligned}
& \Phi(\varepsilon)=-4.41 e^{-\varepsilon / 0.7176} \text { for } \varepsilon>1.9475 \\
& \Phi(\varepsilon)=-1.7817+0.9270 \varepsilon+0.0169 \varepsilon^{2}-0.05148 \varepsilon^{3} \text { for } 0 \leq \varepsilon \leq 1.9475
\end{aligned}
$$

With $\varepsilon=z / b$, where $b \approx 1 \mathrm{fm}$ is the width (diffuseness) of the nuclear surface. The Süsmann central radii $C_{i}$ of the fragments are related to the sharp radii $R_{i}$ as:

$$
C_{i}=R_{i}-\left(\frac{b^{2}}{R_{i}}\right) \mathrm{fm}
$$

For $R_{i}$, we use semi-empirical formula in terms of mass number $A_{i}$ as [19]:

$$
R_{i}=1.28 A_{i}^{1 / 3}-0.76+0.8 A_{i}^{-1 / 3} \mathrm{fm}
$$

The potential for the internal part (overlap region) of the barrier is given as:

$$
V=a_{0}\left(L-L_{0}\right)^{n} \text { for } z<0
$$

Where $L=z+2 C_{1}+2 C_{2}$ fm and $L_{0}=2 C \mathrm{fm}$, the diameter of the parent nuclei. The constants $a_{0}$ and $n$ are determined by the smooth matching of the two potentials at the touching point.

The barrier penetrability $P$ using the one dimensional Wentzel-Kramers-Brillouin approximation, is given as:

$$
P=\exp \left\{-\frac{2}{\hbar} \int_{a}^{b} \sqrt{2 \mu(V-Q)} d z\right\}
$$

Here the mass parameter is replaced by $\mu=m A_{1} A_{2} / A$, where $m$ is the nucleon mass and $A_{1}, A_{2}$ are the mass numbers of daughter and emitted cluster respectively. The turning points " $a$ " and " $b$ " are determined from the equation, $V(a)=V(b)=Q$, where $Q$ is the energy released.

The half-life time is given by:

$$
T_{1 / 2}=\left(\frac{\ln 2}{\lambda}\right)=\left(\frac{\ln 2}{v P}\right)
$$

Here $\lambda$ is the decay constant and $v$ is the assault frequency. The empirical vibration energy $E_{v}$, is given as [8]:

$$
E_{v}=Q\left\{0.056+0.039 \exp \left[\frac{\left(4-A_{2}\right)}{2.5}\right]\right\} \quad \text { for } A_{2} \geq 4
$$

\section{Results and Discussion}

In the present work we have studied various decay modes of superheavy nuclei Ds $(Z=110)$, within the range $256 \leq \mathrm{A} \leq 275$, including proton decay, alpha decay, spontaneous fission and cluster decay. Various theoretical models are used for calculating the decay half-lives.

\subsection{Proton Decay}

The one proton and two proton separation energies of all the isotopes of Ds within the range $256 \leq \mathrm{A} \leq$ 275 are evaluated. The mass excess values taken from Moller et al., [21] have been used for calculating the separation energies. All the possible proton emitters of Ds within $256 \leq \mathrm{A} \leq 275$ are listed in Table 1 . The one proton decay half-lives were calculated using Gamow like model [7]. The half-lives for one proton radioactivity for the isotopes ${ }^{256-260}$ Ds are also shown in TABLE 1. 
Table 1: Probable proton emitters of isotopes of superheavy element Ds within the range $256 \leq \mathrm{A} \leq 275$. The one proton separation energy and two proton separation energy for the isotopes ${ }^{256-262} 110$ are tabulated.

\begin{tabular}{|l|l|l|l|l|}
\hline Parent nuclei & $\mathrm{S}(1 p)$ & $\mathrm{S}(2 p)$ & $\begin{array}{l}\mathrm{Q}_{p} \text { Value } \\
(\mathrm{MeV})\end{array}$ & $T_{1 / 2}^{1 p}(\mathrm{~s})$ \\
\hline${ }^{256} 110$ & -0.881 & -2.752 & 0.881 & $5.422 \mathrm{E}+001$ \\
\hline${ }^{257} 110$ & -0.861 & -2.412 & 0.861 & $2.002 \mathrm{E}+002$ \\
\hline${ }^{258} 110$ & -0.391 & -1.912 & 0.391 & $4.924 \mathrm{E}+026$ \\
\hline${ }^{259} 110$ & -0.351 & -1.542 & 0.351 & $6.899 \mathrm{E}+030$ \\
\hline${ }^{260} 110$ & -0.051 & -1.182 & 0.051 & $2.074 \mathrm{E}+159$ \\
\hline${ }^{261} 110$ & -0.021 & -0.782 & 0.021 & \\
\hline${ }^{262} 110$ & 0.329 & -0.392 & & \\
\hline
\end{tabular}

\subsection{Alpha Decay}

The Q values for the decay are calculated using the mass excess values taken from Moller et al [21]. The alpha decay half-lives of all the isotopes of Ds within the range $256 \leq \mathrm{A} \leq 275$ are calculated using CPPM. For a theoretical comparison the half-lives are also evaluated using Viola-Seaborg semi-empirical relationship (VSS) [22, 23], the Universal curve of Poenaru et al., (UNIV) [17, 24], the analytical formula of Royer [25] and the Universal decay law (UDL) [26, 27]. It is seen that the decay half-lives calculated using CPPM matches well with other theoretical results. The calculations of alpha decay half-lives using CPPM and their comparison with other theoretical models are presented in TABLE 2.

Table 2: The alpha decay half-lives of isotopes of superheavy nuclei $Z=110$ in the range $256 \leq \mathrm{A} \leq 275$. Comparison of the half-lives using 5 different models is given.

\begin{tabular}{|l|l|l|l|l|l|l|}
\hline \multirow{2}{*}{ Parent nuclei } & $Q_{\alpha}(\mathrm{MeV})$ & \multicolumn{2}{l}{$T_{1 / 2}^{\alpha}(\mathrm{s})$} \\
\cline { 3 - 7 } & & $\mathrm{CPPM}$ & VSS & UNIV & Royer & UDL \\
\cline { 2 - 7 } & 12.545 & $4.478 \mathrm{E}-07$ & $1.113 \mathrm{E}-07$ & $1.496 \mathrm{E}-07$ & $1.678 \mathrm{E}-07$ & $8.380 \mathrm{E}-08$ \\
\hline${ }^{256} \mathrm{Ds}$ & 12.455 & $6.661 \mathrm{E}-07$ & $1.949 \mathrm{E}-06$ & $2.109 \mathrm{E}-07$ & $1.069 \mathrm{E}-06$ & $1.243 \mathrm{E}-07$ \\
\hline${ }^{257} \mathrm{Ds}$ & 12.145 & $3.047 \mathrm{E}-06$ & $7.080 \mathrm{E}-07$ & $7.847 \mathrm{E}-07$ & $9.768 \mathrm{E}-07$ & $5.519 \mathrm{E}-07$ \\
\hline${ }^{258} \mathrm{Ds}$ & 12.015 & $5.714 \mathrm{E}-06$ & $1.534 \mathrm{E}-05$ & $1.355 \mathrm{E}-06$ & $7.731 \mathrm{E}-06$ & $1.026 \mathrm{E}-06$ \\
\hline${ }^{259} \mathrm{Ds}$ & 11.885 & $1.083 \mathrm{E}-05$ & $2.478 \mathrm{E}-06$ & $2.365 \mathrm{E}-06$ & $3.125 \mathrm{E}-06$ & $1.928 \mathrm{E}-06$ \\
\hline${ }^{260} \mathrm{Ds}$ & 11.765 & $1.969 \mathrm{E}-05$ & $5.214 \mathrm{E}-05$ & $3.982 \mathrm{E}-06$ & $2.415 \mathrm{E}-05$ & $3.477 \mathrm{E}-06$ \\
\hline${ }^{261} \mathrm{Ds}$ & 11.865 & $1.102 \mathrm{E}-05$ & $2.733 \mathrm{E}-06$ & $2.403 \mathrm{E}-06$ & $3.149 \mathrm{E}-06$ & $1.980 \mathrm{E}-06$ \\
\hline${ }^{262} \mathrm{Ds}$ & 12.015 & $4.781 \mathrm{E}-06$ & $1.534 \mathrm{E}-05$ & $1.163 \mathrm{E}-06$ & $6.527 \mathrm{E}-06$ & $8.785 \mathrm{E}-07$ \\
\hline${ }^{263} \mathrm{Ds}$ & 12.075 & $3.350 \mathrm{E}-06$ & $9.880 \mathrm{E}-07$ & $8.535 \mathrm{E}-07$ & $1.039 \mathrm{E}-06$ & $6.227 \mathrm{E}-07$ \\
\hline${ }^{264} \mathrm{Ds}$ & 11.895 & $8.258 \mathrm{E}-06$ & $2.747 \mathrm{E}-05$ & $1.866 \mathrm{E}-06$ & $1.075 \mathrm{E}-05$ & $1.509 \mathrm{E}-06$ \\
\hline${ }^{265} \mathrm{Ds}$ & 11.705 & $2.196 \mathrm{E}-05$ & $6.043 \mathrm{E}-06$ & $4.367 \mathrm{E}-06$ & $5.824 \mathrm{E}-06$ & $3.942 \mathrm{E}-06$ \\
\hline${ }^{266} \mathrm{Ds}$ & 11.385 & $1.241 \mathrm{E}-04$ & $3.615 \mathrm{E}-04$ & $1.986 \mathrm{E}-05$ & $1.303 \mathrm{E}-04$ & $2.156 \mathrm{E}-05$ \\
\hline${ }^{267} \mathrm{Ds}$ & 11.015 & $1.016 \mathrm{E}-03$ & $2.251 \mathrm{E}-04$ & $1.265 \mathrm{E}-04$ & $1.995 \mathrm{E}-04$ & $1.699 \mathrm{E}-04$ \\
\hline${ }^{268} \mathrm{Ds}$ & 10.685 & $7.219 \mathrm{E}-03$ & $1.672 \mathrm{E}-02$ & $7.201 \mathrm{E}-04$ & $5.551 \mathrm{E}-03$ & $1.169 \mathrm{E}-03$ \\
\hline${ }^{269} \mathrm{Ds}$ & 10.405 & $4.074 \mathrm{E}-02$ & $7.409 \mathrm{E}-03$ & $3.368 \mathrm{E}-03$ & $6.036 \mathrm{E}-03$ & $6.425 \mathrm{E}-03$ \\
\hline${ }^{270} \mathrm{Ds}$ & 10.195 & $1.548 \mathrm{E}-01$ & $3.086 \mathrm{E}-01$ & $1.112 \mathrm{E}-02$ & $9.445 \mathrm{E}-02$ & $2.395 \mathrm{E}-02$ \\
\hline${ }^{271} \mathrm{Ds}$ & 10.055 & $3.807 \mathrm{E}-01$ & $6.339 \mathrm{E}-02$ & $2.492 \mathrm{E}-02$ & $4.743 \mathrm{E}-02$ & $5.820 \mathrm{E}-02$ \\
\hline${ }^{272} \mathrm{Ds}$ & 10.065 & $3.422 \mathrm{E}-01$ & $6.930 \mathrm{E}-01$ & $2.256 \mathrm{E}-02$ & $1.955 \mathrm{E}-01$ & $5.245 \mathrm{E}-02$ \\
\hline${ }^{273} \mathrm{Ds}$ & 10.375 & $4.232 \mathrm{E}-02$ & $8.868 \mathrm{E}-03$ & $3.438 \mathrm{E}-03$ & $6.064 \mathrm{E}-03$ & $6.697 \mathrm{E}-03$ \\
\hline${ }^{274} \mathrm{Ds}$ & 10.775 & $3.284 \mathrm{E}-03$ & $1.000 \mathrm{E}-02$ & $3.499 \mathrm{E}-04$ & $2.598 \mathrm{E}-03$ & $5.415 \mathrm{E}-04$ \\
\hline${ }^{275} \mathrm{Ds}$ & & & &
\end{tabular}

\subsection{Spontaneous Fission}

The spontaneous fission half-lives are calculated using the new shell-effect-dependent formula proposed by Santhosh et al., [13] and is given by:

$$
\log _{10}\left(T_{1 / 2} / y r\right)=a \frac{Z^{2}}{A}+b\left(\frac{Z^{2}}{A}\right)^{2}+c\left(\frac{N-Z}{N+Z}\right)+d\left(\frac{N-Z}{N+Z}\right)^{2}+e E_{\text {shell }}+f
$$

Where $a=-43.25203, b=0.49192, c=3674.3927, d=-9360.6, e=0.8930$ and $f=578.56058 . E_{\text {shell }}$ is the shell correction energy taken from Ref. [21].

The accurate calculations of spontaneous fission half-lives are difficult due to the complexity and uncertainty of fission process. The spontaneous fission half-lives calculated using the new shell dependent formula of Santhosh et al., [13] are compared with the semi-empirical formula of Xu et al. [14], formula proposed by Bao et al. [15], and the formula of Ren et al., [28] and are given in TABLE 3. Model to model variations in the prediction of spontaneous fission half-lives in superheavy region are evident from the table. 
Table 3: The spontaneous fission half-lives of isotopes of superheavy nuclei $Z=110$ in the range $256 \leq A \leq$ 275. Comparison of the half-lives using 4 different models is given.

\begin{tabular}{|c|c|c|c|c|}
\hline \multirow[t]{2}{*}{ Parent nuclei } & \multicolumn{4}{|l|}{$T_{1 / 2}^{S F}$} \\
\hline & KPS & $\mathrm{Xu}$ & Bao & Ren \\
\hline${ }^{256} \mathrm{Ds}$ & $6.787 \mathrm{E}-27$ & $2.896 \mathrm{E}-28$ & $4.955 \mathrm{E}-18$ & $4.617 \mathrm{E}-85$ \\
\hline${ }^{257}$ Ds & $1.031 \mathrm{E}-23$ & $2.835 \mathrm{E}-25$ & $6.369 \mathrm{E}-14$ & $2.064 \mathrm{E}-67$ \\
\hline${ }^{258}$ Ds & $1.084 \mathrm{E}-20$ & $1.837 \mathrm{E}-22$ & $6.772 \mathrm{E}-15$ & $2.864 \mathrm{E}-64$ \\
\hline${ }^{259}$ Ds & $7.486 \mathrm{E}-18$ & $7.876 \mathrm{E}-20$ & $1.290 \mathrm{E}-10$ & $5.204 \mathrm{E}-50$ \\
\hline${ }^{260}$ Ds & $2.679 \mathrm{E}-15$ & $2.236 \mathrm{E}-17$ & $6.317 \mathrm{E}-12$ & $1.970 \mathrm{E}-46$ \\
\hline${ }^{261}$ Ds & $8.216 \mathrm{E}-13$ & $4.202 \mathrm{E}-15$ & $1.407 \mathrm{E}-07$ & $3.081 \mathrm{E}-35$ \\
\hline${ }^{262} \mathrm{Ds}$ & $8.637 \mathrm{E}-11$ & $5.230 \mathrm{E}-13$ & $1.568 \mathrm{E}-09$ & $1.756 \mathrm{E}-31$ \\
\hline${ }^{263} \mathrm{Ds}$ & $6.451 \mathrm{E}-09$ & $4.309 \mathrm{E}-11$ & $3.646 \mathrm{E}-06$ & $4.916 \mathrm{E}-23$ \\
\hline${ }^{264}$ Ds & $2.108 \mathrm{E}-07$ & $2.351 \mathrm{E}-09$ & $9.973 \mathrm{E}-09$ & $2.360 \mathrm{E}-19$ \\
\hline${ }^{265}$ Ds & $7.513 \mathrm{E}-06$ & $8.497 \mathrm{E}-08$ & $2.693 \mathrm{E}-05$ & $2.418 \mathrm{E}-13$ \\
\hline${ }^{266} \mathrm{Ds}$ & $1.144 \mathrm{E}-04$ & $2.034 \mathrm{E}-06$ & 7.309E-08 & $5.534 \mathrm{E}-10$ \\
\hline${ }^{267}$ Ds & $2.371 \mathrm{E}-03$ & $3.225 \mathrm{E}-05$ & $4.259 \mathrm{E}-04$ & $4.174 \mathrm{E}-06$ \\
\hline${ }^{268} \mathrm{Ds}$ & $2.365 \mathrm{E}-02$ & $3.387 \mathrm{E}-04$ & $3.678 \mathrm{E}-06$ & $2.611 \mathrm{E}-03$ \\
\hline${ }^{269}$ Ds & $3.472 \mathrm{E}-01$ & $2.357 \mathrm{E}-03$ & $8.604 \mathrm{E}-02$ & $2.869 \mathrm{E}-01$ \\
\hline${ }^{270} \mathrm{Ds}$ & $1.868 \mathrm{E}+00$ & $1.087 \mathrm{E}-02$ & $1.003 \mathrm{E}-03$ & $2.844 \mathrm{E}+01$ \\
\hline${ }^{271}$ Ds & $1.441 \mathrm{E}+01$ & $3.319 \mathrm{E}-02$ & $2.707 \mathrm{E}+01$ & $8.872 \mathrm{E}+01$ \\
\hline${ }^{272} \mathrm{Ds}$ & $4.051 \mathrm{E}+01$ & $6.720 \mathrm{E}-02$ & $3.367 \mathrm{E}-01$ & $8.183 \mathrm{E}+02$ \\
\hline${ }^{273}$ Ds & $8.759 \mathrm{E}+01$ & $9.016 \mathrm{E}-02$ & $8.690 \mathrm{E}+02$ & $1.390 \mathrm{E}+02$ \\
\hline${ }^{274} \mathrm{Ds}$ & $4.186 \mathrm{E}+01$ & $8.017 \mathrm{E}-02$ & $1.477 \mathrm{E}-01$ & $7.079 \mathrm{E}+01$ \\
\hline${ }^{275}$ Ds & $1.529 \mathrm{E}+01$ & $4.725 \mathrm{E}-02$ & $4.818 \mathrm{E}+00$ & $1.239 \mathrm{E}+00$ \\
\hline
\end{tabular}

\subsection{Cluster Decay}

Recently much attention has been received for the emission of clusters heavier than alpha particles. The cluster decay half-lives for the emission of clusters ${ }^{8,10} \mathrm{Be},{ }^{12,14} \mathrm{C},{ }^{16,18,20,22} \mathrm{O},{ }^{20,22,24,26} \mathrm{Ne},{ }^{24,26,28,30} \mathrm{Mg}$ from all the isotopes of Ds within the range $256 \leq \mathrm{A} \leq 275$ has been studied using CPPM. The Q values for the decay are calculated using the mass excess values taken from Moller et al [21]. For those clusters whose mass excess values are not available in Moller et al., the corresponding values are taken from Wang et al [29]. Fig 1. Shows the plot for $\log _{10} \mathrm{~T}_{1 / 2}$ versus mass number for all the possible cluster emission including the alpha particle emission from the isotopes ${ }^{256-275}$ Ds. Experimental upper and lower limits are also marked in the figure using dotted black lines.

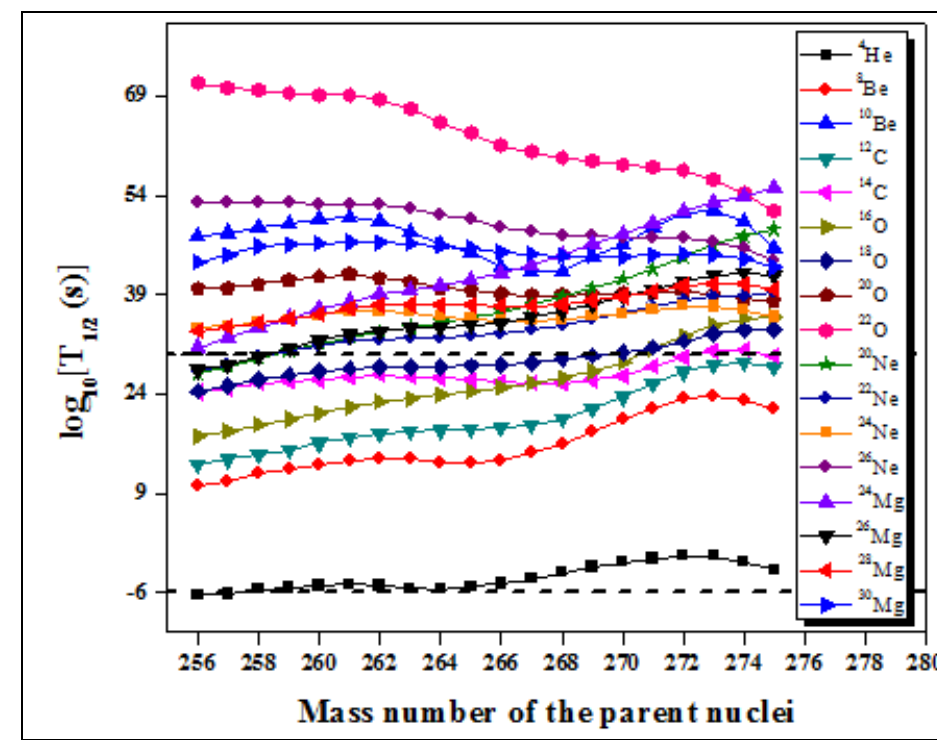

Fig 1: Plot for the emission of clusters, ${ }^{4} \mathrm{He},{ }^{8,10} \mathrm{Be},{ }^{12,14} \mathrm{C},{ }^{16,18,20,22} \mathrm{O},{ }^{20,22,24,26} \mathrm{Ne},{ }^{24,26,28,30} \mathrm{Mg}$, from ${ }^{256-275} \mathrm{Ds}$.

\section{Conclusion}

Knowledge of decay properties is inevitable in the study of superheavy nuclei. The decay modes of superheavy nuclei includes proton decay, alpha decay, spontaneous fission and cluster decay. A theoretical 
analysis on all these four decay modes has been performed in this paper by considering the isotopes of superheavy nuclei Ds in the region $256 \leq \mathrm{A} \leq 275$. Various theoretical models are used for calculating the halflive for proton decay, alpha decay, spontaneous fission and cluster decay. The study shows that most of the isotopes of superheavy nuclei will decay through alpha emission. Besides, proton decay, spontaneous fission and cluster decay are also possible in the region of superheavy nuclei.

\section{References}

[1] W. Myers, and W. Swiatecki, Nuclear masses and deformations, Nuclear Physics, 81(2), 1966, 1-60.

[2] U. Mosel, and W. Greiner, On the stability of superheavy nuclei against fission, Z.Physik, 222(3), 1969, 261-282.

[3] Yu. Ts. Oganessian, and V K Utyonkov, Super-heavy element research, Reports on Progress in Physics, 78(3), 2015, 036301-036322.

[4] S. Hofmann, and G. Munzenberg, The discovery of the heaviest elements, Reviews of Modern Physics, 72(3), 2000, 733-767.

[5] S. Aberg, P. B. Semmes, and W. Nazarewicz, Spherical proton emitters, Physical Review C, 56(4), 1997, 1762-1773.

[6] S. B. Duarte, O. A. P. Tavares, F. Guzman, A. Dimarco, F. Garcia, O. Rodriguez, and M. Goncalves, Half-lives for proton emission, alpha decay, cluster radioactivity, and cold fission processes calculated in a unified theoretical framework, Data and Nuclear Data Tables, 80(2), 2002, 235-299.

[7] A. Zdeb, M.Warda, C.M. Petrache, and K. Pomorski, Proton emission half-lives within a Gamow-like model, The European Physical Journal A, 52(10), 2016, 323-329.

[8] D. N. Poenaru, M.Ivascu, A. Sandulescu, and W. Greiner, Atomic nuclei decay modes by spontaneous emission of heavy ions Physical Review C, 32(2), 1985, 572-581.

[9] B. Buck and A. C. Merchant, and S. M. Perez, $\alpha$ decay calculations with a realistic potential, Physical Review C, 45(5), 1992, 2247-2253.

[10] D. N. Basu, Role of effective interaction in nuclear disintegration process, Physics Letters B, 566(1), 2003, 90-97.

[11] J. C. Pei, F. R. Xu, Z. J. Lin, and E. G. Zhao, $\alpha$-decay calculations of heavy and superheavy nuclei using effective mean-field potentials, Physical Review C, 76(4), 2007, 044326-044334.

[12] R. Smolanczuk, J. Skalski, and A. Sobiczwski, Spontaneous fission half lives of deformed superheavy nuclei, Physical Review C, 52(4), 1995, 1871-1880.

[13] K. P. Santhosh, and C. Nithya, Theoretical studies on the modes of decay of superheavy nuclei, Physical Review C, 94(5), 2016, 054621-054630.

[14] C. Xu, Z. Ren, and Y. Guo, Competition between $\alpha$ decay and spontaneous fission for heavy and superheavy nuclei, Physical Review C, 78(4), 2008, 044329-044336.

[15] X. J. Bao, S. Q. Guo, H. F. Zhang, Y. Z. Xing, J. M. Dong, and J. Q. Li, Competition between $\alpha$-decay and spontaneous fission for superheavy nuclei, Journal of Physics G: Nuclear and Particle Physics, 42(8), 2015, 085101085114.

[16] D. N. Poenaru, R. A. Gherghescu, and W. Greiner, Heavy-particle radioactivity of superheavy nuclei, Physical Review Letters, 107(6), 2011, 062503-062506.

[17] D. N. Poenaru, R. A. Gherghescu, and W. Greiner, Cluster decay of superheavy nuclei, Physical Review C, 85(3), 2012, 034615-034621.

[18] K. P. Santhosh, and A. Joseph, Cluster radioactivity in xenon isotopes, Pramana, 62(4), 2004, 957-965.

[19] J. Blocki, J. Randrup, W. J. Swiatecki, and C. F. Tsang, Proximity Forces, Annals of Physics 105(2), 1977, 427-462.

[20] J. Blocki, and W. J. Swiatecki, A generalization of the proximity force theorem, Annels of Physics, 132(1), 1981, 5365.

[21] P. Moller, A. J. Sierk, T. Ichikawa, and H. Sagawa, Nuclear ground-state masses and deformations, Atomic Data and Nuclear Data Tables, 109, 2016, 1-204.

[22] V. E. Viola. Jr,. and G. T. Seaborg, Nuclear systematic of the heavy elements-II life times for alpha, beta and spontaneous fission decay, Journal of Inorganic Nuclear Chemistry, 28(3), 1966, 741-761.

[23] A. Sobiczewski, Z. Patyk, and S. Cwiok, Deformed superheavy nuclei, Physics Letters B, 224(1-2), 1989, 1-4.

[24] D. N. Poenaru, R. A. Gherghescu, and W. Greiner, Single universal curve for cluster radioactivities and $\alpha$ decay, Physical Review C, 83(1), 2011, 014601-014604.

[25] G. Royer, Journal of Physics G: Nuclear and Particle Physics, 26(8), 2000, 1149-1170.

[26] C. Qi, F. R. Xu, R. J. Liotta, R. Wyss, M. Y .Zhang, C. Asawatangtrakuldee, and D. Hu, Microscopic mechanism of charged-particle radioactivity and generalization of the Geiger-Nuttall law, Physical Review C, 80(4), 2009, 044326044334.

[27] C. Qi, F.R. Xu, R.J. Liotta, and R. Wyss, Universal decay law in charged-particle emission and exotic cluster radioactivity, Physical Review Letters, 103(7), 2009, 072501-072504.

[28] Z. Ren, and C. Xu, Spontaneous fission half-lives of heavy nuclei in ground state and in isomeric state, Nuclear Physics A, 759(1), 2005, 64-78.

[29] M. Wang, G. Audi, A. H. Wapstra, F. G. Kondev, M. Mac Cormic, X. Xu, and B. Pfeiffer, Chinese Physics C, $36(12), 2012,1603-2014$. 